OS-GE-39-93

October 1993

\title{
Quantum Group Symmetry and Quantum Hall Wavefunctions on a Torus
}

\author{
Haru-Tada Sato ${ }^{\dagger}$ \\ Institute of Physics, College of General Education \\ Osaka University, Toyonaka, Osaka 560, Japan
}

\begin{abstract}
We find a quantum group structure in two-dimensional motion of nonrelativistic electrons in a uniform magnetic field on a torus. The representation basis of the quantum algebra is composed of the quantum Hall wavefunctions proposed by Haldane-Rezayi at the Landau-level filling factor $\nu=1 / m$ ( $m$ odd). It is also shown that the quantum group symmetry is relevant to the degenerate Landau states and the deformation parameter of the quantum algebra is given by the filling factor.
\end{abstract}

$\dagger$ Fellow of the Japan Society for the

Promotion of Science

E-mail address : hsato@jpnyitp. yukawa.kyoto-u.ac.jp 
There have been many discussions in the study of quantum groups (quantum universal enveloping algebras [1, 2]). Quantum group structures are found in (2+1)-dimensional topological Chern-Simons theories [3] as well as in rational conformal field theories and integrable lattice models [4]. Although the abelian Chern-Simons theory does not possess a quantum group structure in the literature [3], it might be possible to exhibit one in some other senses. There have been also interesting investigations of condensed matter problems such as the fractional quantum Hall effect [5] making use of the abelian ChernSimons theory.

We have considered the two-dimensional planer motion of a nonrelativistic particle in a uniform magnetic field in the previous paper [12] and we derived the quantum group algebra $\mathcal{U}_{q}(\operatorname{sl}(2))$ acting within each Landau level. It can be understood from the result of that paper that the wavefunctions form a representation basis of the $\mathcal{U}_{q}(s l(2))$ algebra and that the deformation parameter $q$ is related to the filling factor $\nu=1 /(2 j+1)$ by $q=\exp (2 \pi i \nu)$. These observations motivate us to discuss a relation between quantum groups and the quantum Hall effects.

In this report we consider a $N_{e}$-electron system in the lowest Landau level on a square torus of side $L$. We show that this case also exhibits a quantum group symmetry similarly as the planer case and the deformation parameter $q$ is given by the filling factor $\nu=1 / m$ ( $m$ odd integer).

We use the results of the previous paper and thus start with reviewing some formulae. In the system of a charged particle in a constant magnetic field $B$ perpendicular to the $x-y$ plane, the generators of the quantum group algebra are realized by the magnetic translation operators $T_{\alpha}$ [7]

$$
T_{\left(\alpha_{1}, \alpha_{2}\right)}=\exp \left(\frac{i}{\hbar} \alpha \cdot \beta\right)
$$

where

$$
\begin{gathered}
\beta_{i}=p_{i}-\frac{e}{c} A_{i}-\frac{e B}{c} \epsilon_{i j} x^{j} \\
\epsilon_{11}=\epsilon_{22}=0, \quad \epsilon_{12}=-\epsilon_{21}=1
\end{gathered}
$$


and the gauge potential is

$$
A_{i}=-\frac{1}{2} B \epsilon_{i j} x^{j}-\partial_{i} \Lambda
$$

The vector $\beta$ is related to the cyclotron center and we choose the scalar function $\Lambda$ to be $\Lambda=\frac{1}{2} B x y$ for simplicity. The following combinations of magnetic translations [12]

$$
\begin{gathered}
E^{+}=\frac{T_{(\Delta, \bar{\Delta})}-T_{(-\Delta, \bar{\Delta})}}{q-q^{-1}} \\
E^{-}=\frac{T_{(-\Delta,-\bar{\Delta})}-T_{(\Delta,-\bar{\Delta})}}{q-q^{-1}} \\
k=T_{(\Delta, 0)} .
\end{gathered}
$$

satisfy the defining relations of the $\mathcal{U}_{q}(s l(2))$

$$
\left[E^{+}, E^{-}\right]=\frac{k^{2}-k^{-2}}{q-q^{-1}}, \quad k E^{ \pm} k^{-1}=q^{ \pm 1} E^{ \pm}
$$

with the identification

$$
q=\exp \left(i \Delta \bar{\Delta} l_{B}^{-2}\right) .
$$

The quantity $l_{B}$ is called a magnetic length $l_{B}=\sqrt{\hbar c / e B}$. If we put $\Delta=L_{x} /(2 j+1)$ and $\bar{\Delta}=L_{y} /(2 j+1)$, the Landau states $\psi_{l}(-j \leq l \leq j)$ [6] behave as a spin- $j$ representation of $\mathcal{U}_{q}(s l(2))$. Namely, we obtain the relatons 12

$$
\begin{gathered}
E^{ \pm} \psi_{l}=\left[\frac{1}{2} \pm l\right]_{q} \psi_{l \pm 1}, \\
k \psi_{l}=q^{l} \psi_{l},
\end{gathered}
$$

where the notation $[x]_{q}$ means

$$
[x]_{q}=\frac{q^{x}-q^{-x}}{q-q^{-1}} .
$$

We note that $k$ measures the quantum number $l$ and $E^{+}\left(E^{-}\right)$raises (lowers) the $l$ and that our quantum algebra is associated with only the quantum number $l$, namely the degeneracy of the Landau levels. This means that the energy level (the Landau level) is invariant under the action of the quantum algebra. This is the difference from the case of the $s u(2)$ angular momentum algebra. 
Now we generalize the above argument to the case of a torus. The quantum Hall wavefunctions on a torus are proposed by Haldane and Rezayi [ [ $[$ ] and discussed in some other papers [9, 10]. Our aim is to examine whether the similar relation as (10) and (11) holds when we operate the generators (5)-(7) to the wavefunction basis. First, we consider the one-particle system on a torus in the lowest Landau level. For simplicity, we specify the case of a square torus of side $L$ in the unit $l_{B}=1$ and the gauge $\Lambda=\frac{1}{2} B x y$ [9, 11]. The lowest Landau-level wavefunction is written as

$$
\psi=\exp \left(-\frac{1}{2} y^{2}\right) f(z)
$$

where $f(z)$ is an analytic function of $z=x+i y$ which satisfies the periodicity condition

$$
f(z)=e^{-\frac{1}{2} L^{2}} e^{i L z} f(z+i L) .
$$

If the total flux $N_{s}$ through the surface of the torus satisfies the condition

$$
L^{2}=2 \pi N_{s},
$$

we have $N_{s}$ linearly independent solutions of (14) [G]

$$
f_{l}(z)=\Theta\left[\begin{array}{c}
\frac{l}{N_{s}} \\
0
\end{array}\right]\left(\frac{N_{s}}{L} z \mid i N_{s}\right), \quad l=1, \ldots, N_{s}
$$

where

$$
\Theta\left[\begin{array}{l}
a \\
b
\end{array}\right](w \mid \tau)=\sum_{n \in \mathbf{Z}} \exp \left\{i \pi \tau(n+a)^{2}+2 \pi i(n+a)(w+b)\right\} .
$$

For the convenience of notations, let us introduce the following magnetic translations [1]

$$
S_{(n, m)} \equiv T_{(a n, a m)}, \quad a=\frac{L}{N_{s}},
$$

With the use of (15), we can verify that these operators and the wavefuctions

$$
\psi_{l}=\exp \left(-\frac{1}{2} y^{2}\right) f_{l}(z)
$$

satisfy the following relations

$$
\begin{gathered}
S_{(n, 0)} \psi_{l}(z)=e^{2 \pi i \frac{l}{N_{s}} n} \psi_{l}(z), \\
S_{(0, n)} \psi_{l}(z)=\psi_{l+n}(z),
\end{gathered}
$$


and the multiplication law

$$
S_{(n, m)}=e^{-\frac{i}{2} n m a^{2}} S_{(n, 0)} S_{(0, m)} .
$$

Combining (20), (21) and (15), we get

$$
S_{(n, m)} \psi_{l}(z)=\exp \left[2 \pi i \frac{n}{N_{s}}\left(\frac{m}{2}+l\right)\right] \psi_{l+n}(z) .
$$

Putting $\Delta=a n, \bar{\Delta}=a m$ in (5)-(9), we easily recognize that

$$
E^{+}=\frac{S_{(n, m)}-S_{(-n, m)}}{q-q^{-1}}, \quad E^{-}=\frac{S_{(-n,-m)}-S_{(n,-m)}}{q-q^{-1}}, \quad k=S_{(n, 0)}
$$

satisfy the $\mathcal{U}_{q}(\operatorname{sl}(2))$ algebra (8) with

$$
q=\exp \left(i a^{2} n m\right)
$$

We thus obtain the action of the quantum algebra on the torus

$$
\begin{gathered}
E^{ \pm} \psi_{l}(z)=\frac{\exp \left[2 \pi i \frac{n}{N_{s}}\left(\frac{m}{2} \pm l\right)\right]-\exp \left[-2 \pi i \frac{n}{N_{s}}\left(\frac{m}{2} \pm l\right)\right]}{q-q^{-1}} \psi_{l+m}(z), \\
k \psi_{l}(z)=\exp \left(2 \pi i \frac{n}{N_{s}} l\right) .
\end{gathered}
$$

We consequently have the same equations as (10) and (11) when we put $n=m=1$ in the above equations (25). It can be seen from (24) that the value of $q$ is related to the filling factor

$$
\nu \equiv \frac{N_{e}}{N_{s}}
$$

by the relation

$$
q=\exp (2 \pi i \nu)
$$

The above case corresponds to $N_{e}=1$ and the other cases $\left(N_{e} \neq 1\right)$ are discussed below.

Next we discuss the case of $N_{e}$-electron wavefunctions. Needless to say in the noninteracting particle system, the comultiplication law of the quantum algebra gives rise to the quantum group generators on the tensor product representations of each particle's wavefunction;

$$
E^{ \pm}=\sum_{i=1}^{N_{e}} k_{1} \ldots k_{i-1} E_{i}^{ \pm} k_{i+1}^{-1} \ldots k_{N_{e}}^{-1}, \quad k=\prod_{i=1}^{N_{e}} k_{i}
$$


and

$$
\psi_{l_{1}, \ldots, l_{N_{e}}}=\otimes_{i=1}^{N_{e}} \psi_{l_{i}}\left(x_{i}, y_{i}\right)
$$

On the other hand, our intereset being a system with the filling factor given by

$$
\nu=\frac{1}{m} \quad(m \text { odd integer })
$$

we work with the wavefunctions of interacting particle system on the torus in Ref. [11]

$$
\begin{gathered}
\psi_{l}=\exp \left[-\frac{1}{2} \sum_{i=1}^{N_{e}} y_{i}^{2}\right] f_{l}\left(z_{1}, \ldots, z_{N_{e}}\right), \\
f_{l}\left(z_{1}, \ldots, z_{N_{e}}\right)=\prod_{j<k}^{N_{e}}\left[\frac{\Theta_{1}\left(z_{j}-z_{k} \mid i\right)}{\Theta_{1}(0 \mid i)}\right]^{m} \Theta\left[\begin{array}{c}
\frac{l}{m} \\
0
\end{array}\right]\left(\frac{m}{L} \sum_{j} z_{j} \mid i m\right),
\end{gathered}
$$

where the function $\Theta_{1}$ is the elliptic theta function. Similarly to the above case of the oneparticle system, we introduce the magnetic translation operators for the multi-particle system and operate them on the wavefunctions (31). The total magnetic translation operator is defined by the products of the $N_{e}$ copies of the one-particle magnetic translation

$$
S_{(n, m)}^{t o t}=\prod_{i=1}^{N_{e}} S_{(n, m)}^{(i)}\left(x_{i}, y_{i}\right)
$$

We then have the following relations instead of (20)

$$
\begin{gathered}
S_{(n, 0)}^{t o t} \psi_{l}(z)=e^{2 \pi i \frac{N_{e}}{N_{s}} l n} \psi_{l}(z) \\
S_{(0, n)}^{t o t} \psi_{l}(z)=\psi_{l+n}(z)
\end{gathered}
$$

and the multiplication formula for $S^{\text {tot }}$ owing to (21)

$$
S_{\left(n_{1}, n_{2}\right)}^{t o t} S_{\left(m_{1}, m_{2}\right)}^{t o t}=\exp \left(\frac{i}{2} a^{2} N_{e} \epsilon^{i j} n_{i} m_{j}\right) S_{\left(n_{1}+n_{2}, m_{1}+m_{2}\right)}^{t o t} .
$$

The generators of the quantum group algebra are obtained by replacing $S$ with $S^{\text {tot }}$ in (23) and the commutation relations (8) are checked using the above formula (35) when we set

$$
q=\exp \left(i a^{2} N_{e} n m\right)
$$


We thus find the similar equations as (25)

$$
\begin{gathered}
E^{ \pm} \psi_{l}(z)=\frac{\exp \left[2 \pi i \nu n\left(\frac{m}{2} \pm l\right)\right]-\exp \left[-2 \pi i \nu n\left(\frac{m}{2} \pm l\right)\right]}{q-q^{-1}} \psi_{l+m}(z) \\
k \psi_{l}(z)=\exp (2 \pi i \nu l)
\end{gathered}
$$

Again putting $n=m=1$, we verify that (36) coincides with (27) and the equations (37) become the same relations as (10) and (11).

In this letter we showed that the quantum Hall wavefunctions on a torus form a representation basis of the quantum algebra $\mathcal{U}_{q}(\operatorname{sl}(2))$ of which deformation parameter $q$ is related to the filling factor $\nu=1 / m$ ( $m$ odd integer) by the relation $q=\exp (2 \pi i \nu)$, i.e., (27). This statement is supported also by the result of Ref. 12 which referred to the planer one-particle case. It is interesting to note that the relation between $q$ and $\nu$ appears in accordance with $q$ being a $m$-th root of unity. We have discussed only the case of a primitive $m$-th root of unity, however we should investigate into other $m$-th

root cases in order to completely understand the relation (27) and the relevance of the quantum group structure to the quantum Hall systems. Furthermore it is speculated that the representation theory of quantum groups is expected to give a new approach to the quantum Hall effects as well as to some other topics of the two-dimensional electrons such as anyon systems [13].

\section{Acknowledgments}

The author would like to thank N. Aizawa for a useful suggestion. 


\section{References}

[1] V. Drinfeld, Proc. ICM-86, Berkeley, 1986, pp.798-820;

M. Jimbo, Lett. Math. Phys. 10 (1985) 63; 11 (1986) 247.

[2] P. P. Kulish and N. Yu. Reshetikhin, J. Sov. Math. 23 (1983)

E. K. Sklyanin, Usp. Math. Nauk. 40 (1985) 214.

[3] E. Witten, Commun. Math. Phys. 121 (1989) 351;

G. Siopsis, Mod. Phys. Lett. A6 (1991) 1515;

E. Guadagnini, M. Martellini and M. Mintchev, Nucl. Phys. B336 (1990) 581.

[4] "Yang-Baxter Equation in Integrable Systems", ed. M. Jimbo (World Scientific, Singapore, 1990).

[5] "The Quantum Hall Effect", ed. R. E. Prange and S. M. Girvin (Springer-Verlag, New York, 1986).

[6] L. Landau and E. Lifshitz, "Quantum Mechanics", 3rd ed. (Pergamon, Oxford, 1977).

[7] E. Brown, Phys. Rev. 133 (1964) A1038;

J. Zak, Phys. Rev. 134 (1964) A1602; ibid. A1607.

[8] F.D.M. Haldane and E.H. Rezayi, Phys. Rev. B31 (1985) 2529.

[9] R.B. Laughlin, Ann. Phys. 191 (1989) 163.

[10] G. Cristofano, G. Maiella, R. Musto and F. Nicodemi, Phys. Lett. B262 (1991) 88.

[11] G. Cristofano, G. Maiella, R. Musto and F. Nicodemi, Mod. Phys. Lett. A6 (1991) 1779; ibid. 2985.

[12] H.-T. Sato, "Landau Levels and Quantum Group", Osaka Univ. preprint OS-GE36-93. 
[13] A. Lerda and S. Sciuto, Nucl. Phys. B401 (1993) 613;

R. Caracciolo and M.A.R. Monteiro, Phys. Lett. B308 (1993) 58. 